\title{
Commonly used bowel preparations have significant and different effects upon cell proliferation in the colon: a pilot study Lisa J Croucher*1, Jonathan P Bury ${ }^{2}$, Elizabeth A Williams ${ }^{1}$, Stuart A Riley ${ }^{3}$ and Bernard M Corfe ${ }^{1}$
}

Address: ${ }^{1}$ Human Nutrition Unit, School of Medicine and Biomedical Sciences, University of Sheffield, Sheffield, UK, ${ }^{2}$ Academic Unit of Pathology, School of Medicine and Biomedical Sciences, University of Sheffield, Sheffield, UK and ${ }^{3}$ Department of Gastroenterology, Northern General Hospital, Sheffield, UK

Email: Lisa J Croucher* - L.Croucher@arc.org.uk; Jonathan P Bury - J.P.Bury@sheffield.ac.uk;

Elizabeth A Williams - E.A.Williams@sheffield.ac.uk; Stuart A Riley - Stuart.Riley@sth.nhs.uk; Bernard M Corfe - B.M.Corfe@sheffield.ac.uk

* Corresponding author

Published: 14 November 2008

BMC Gastroenterology 2008, 8:54 doi:10.1 I86/I47|-230X-8-54

This article is available from: http://www.biomedcentral.com/I47I-230X/8/54

(c) 2008 Croucher et al; licensee BioMed Central Ltd.

This is an Open Access article distributed under the terms of the Creative Commons Attribution License (http://creativecommons.org/licenses/by/2.0), which permits unrestricted use, distribution, and reproduction in any medium, provided the original work is properly cited.
Received: 19 May 2008

Accepted: 14 November 2008

\begin{abstract}
Background: Markers of crypt cell proliferation are frequently employed in studies of the impact of genetic and exogenous factors on human colonic physiology. Human studies often rely on the assessment of tissue acquired at endoscopy. Modulation of cell proliferation by bowel preparation with oral laxatives may confound the findings of such studies, but there is little data on the impact of commonly used bowel preparations on markers of cell proliferation.
\end{abstract}

Methods: Crypt length, crypt cellularity and crypt cell proliferation were assessed in biopsies acquired after preparation with either Klean-Prep or Picolax. Crypt cell proliferation was assessed by whole-mount mitotic figure count, and by two different immunohistochemical (IHC) labelling methods (Ki-67 and $\mathrm{pHH} 3$ ). Subsequent biopsies were obtained from the same patients without bowel preparation and similarly assessed. Parameters were compared between groups using analysis of variance and paired $t$-tests.

Results: There were significant differences in labelling indices (LI) between biopsies taken after Klean-prep and those taken after Picolax preparation, for both Ki67 $(\mathrm{p}=0.019)$ and $\mathrm{pHH} 3(\mathrm{p}=$ 0.017 ). A similar trend was seen for whole-mount mitotic figure counts. Suppression or elevation of proliferation parameters by bowel preparation may mask any effect due to an intervention or disease.

Conclusion: Commonly used bowel preparations may have significant and different effects on crypt cell proliferation. This should be taken into account when designing studies and when considering the findings of existing studies.

\section{Background}

Oral laxatives are routinely used to cleanse the colon prior to colonoscopy, to permit clear visualisation of the colonic mucosa and minimise the risk of infection.
Hyperosmotic preparations such as sodium phosphate $(\mathrm{NaP})$ and Picolax ${ }^{\otimes}$ actively draw water into the bowel lumen, increasing stool bulk and stimulating gut motility[1]. The effect is lavage with rapid transit and evacua- 
tion of bowel contents. The fluid and electrolyte imbalance that may occur with preparations of this type precludes their use in patients with renal or congestive heart failure, but they are otherwise well tolerated[2]. Klean-Prep ${ }^{\varpi}$ is principally a lavage solution with some osmotic activity, the large ingestion volume being retained within the colon by polyethelene glycol (PEG), itself relatively inert and poorly absorbed. Although the risk of significant fluid or electrolytes shifts is reduced with PEG based preparations[3], the large ingestion volume can be associated with poor patient compliance[4].

Ideally, a bowel preparation agent should have minimal impact on the colonic mucosa. However, endoscopic and histological abnormalities have been reported, particularly with $\mathrm{NaP}$, and include inflammation, haemorrhage, focal active colitis (FAC), erosion of the surface epithelium and apthoid ulceration [5-7]. One study reported apthoid lesions in $24.5 \%$ of patients prepared with $\mathrm{NaP}$, compared to only $2.3 \%$ of patients prepared with PEG and concluded that NaP should not be used to prepare patients with suspected inflammatory bowel disease[8]. Another study attributing FAC and aphthoid ulcers to $\mathrm{NaP}$ found no lesions in a subset of patients subsequently reexamined without preparation[9]. Sodium picosulfate, the active constituent of Picolax, has been shown to increase the expression of acidic mucin and cytokeratin AE1 in rat colonocytes[10]. The effects of PEG on the morphology of the colonic mucosa are less clear. No significant changes were attributed to PEG in two studies of human and rat colon[6,7]. However, subtle structural changes including mucin depletion, epithelial cell loss and inflammatory cell infiltration of the mucosa have been reported elsewhere[5].

Cell proliferation is a commonly used end-point in studies of the effects of exogenous and genetic factors on colonic mucosal function and cancer risk. However, data on the impact of bowel preparation agents on cell proliferation is sparse. A $136 \%$ increase in colonocyte Ki-67 labelling index has been reported in patients prepared with $\mathrm{NaP}$, when compared to the same patients, unprepared[9]. Sodium picosulfate was found in an animal study to have no effect on colonocyte proliferation[11], whilst another detected increased proliferation in response to bisocodyl and sennosides, laxatives with a similar action[12].

To date, there are no published studies examining or comparing the direct effects of sodium picosulfate and PEG on colonocyte proliferation. This study aims to compare the effects of these two commonly used bowel preparative agents on immunohistochemical and whole-mount derived indices of cell proliferation.

\section{Methods \\ Ethical approval and patient groups}

Patients attending endoscopy clinics at the Northern General Hospital, Sheffield for an initial colonoscopy were recruited to the study. Patients were all male, over 40 and had normal bowel health. Smokers and diabetics were excluded. Patients prepared for endoscopy with KleanPrep $(n=4)$ or Picolax $(n=3)$ were invited to return four weeks later for sigmoidoscopy without bowel preparation. Two biopsies were taken at the recto-sigmoid junction. This study was approved by the North Sheffield Research Ethics Committee (Reference number: 06/ Q2308/93). Informed consent was obtained from all patients.

\section{Measurement of crypt cell proliferation}

The range of markers currently utilised for the assessment of colonocyte proliferation is wide, and may in part account for the variability seen between studies. In this study we employed two recognised immunohistochemical markers of cell proliferation, $\mathrm{Ki}-67$ and pHH3. Ki-67 is expressed throughout the cell cycle, whilst pHH3 (phosphorylated histone $\mathrm{H} 3$ ) is detectable in increasing quantity during the progression from from interphase to prophase. This correlates with a progression from granular to diffuse immunohistochemical staining patterns[13]. Counts for both staining patterns were recorded. Where possible, an additional biopsy from each site was taken for whole-crypt mitotic count, assessed using the microdissection method described by Goodlad, et. al[14].

\section{Immunohistochemical Analyses}

Biopsies were fixed for 24 hours in formalin before paraffin embedding and cutting of serial 4-micron sections at 40-micron intervals. Endogenous peroxidise activity was blocked with $2 \%$ hydrogen peroxide. Heat-induced eiptope retrieval (HIER) was performed using a sodium citrate buffer ( $\mathrm{pH}$ 6.0) for Ki-67 and EDTA ( $\mathrm{pH}$ 8.0) for pHH3. Non-specific immunoglobulin binding sites were blocked with normal horse serum. Slides were incubated for 1 hour with primary antibodies to Ki-67 (Vector Laboratories VP-K 452) and pHH3 (Upstate 06-570) at 1:100 and 1:200, respectively. Staining was visualised with the Vectastain Universal Elite kit and DAB peroxidise substrate (Vector Laboratories). Sections were counterstained with Gill's haematoxylin.

A maximum of 10 well-orientated hemi-crypts per biopsy, showing the entire length of the crypt wall from the base abutting the muscularis mucosa through to the junction with the surface epithelium, were included. Images were captured at 20× magnification with a Nikon D5-M camera at $2560 \times 1920$ resolution, stored without compression and analysed using Nikon NIS-Elements D (v 2.30) software. All cell counts and measurements were made by one 
observer blind to the status of the biopsy. A subset of scores was confirmed by a second independent observer.

The number of cells showing positive nuclear staining for each antigen was recorded as a fraction of the total cell count per hemi-crypt (labelling index:LI). For pHH3, the LIs for granular and diffuse staining were recorded separately. Crypt lengths for up to five crypts per biopsy were determined.

\section{Whole mount analysis}

Whole biopsies were fixed in Carnoy's fluid, then stored in $70 \%$ ethanol. Rehydration through $50 \%$ and $25 \%$ alcohols and PBS was followed by hydrolysis in $5 \mathrm{M} \mathrm{NaOH}$ at room temperature. After staining with Schiff's reagent for 90 minutes, the biopsies were transferred to $45 \%$ acetic acid for dissection. Small groups of crypts were isolated from surrounding tissue under a dissecting microscope, then transferred to slides and squashed with thumb pressure under a cover-slip with aqueous mountant. Mitoses were counted at $\times 40$ in up to ten whole crypts per biopsy, through the full crypt depth. Nuclei in all phases of mitosis were counted and expressed as the number of mitoses per crypt.

\section{Statistics}

Statistical analysis was carried out with SPSS (version 11.0). The distribution of values for each parameter was tested for normality using the Kolmorogov-Smirnov test. Indices for paired biopsies (i.e those from the same patient, with and without preparation) were compared using the paired Student t-test. One-way ANOVA was used to test for the equality of the means for each parameter between each group (no preparation, Klean-Prep and Picolax), and the unpaired t-test was used to test for differences between biopsies taken after Klean-prep and those taken after Picolax.

\section{Results and discussion Proliferation indices}

Immunohistochemical methods for the assessment of cell proliferation in small biopsies suffer from a number of potential disadvantages. Firstly, it can be difficult to obtain high yields of assessable crypts in histological sections. Secondly, there is concern over the impact of the so called "denominator effect" on the LI. Counting mitotic figures in whole-mount preparations avoids these issues, but does suffer the disadvantage that a second biopsy must be obtained if conventional histolological examination (or additional immunohistochemical staining, e.g. for apoptosis) is required. We compared the statistical reliability of both approaches using the Cronbach's alpha statistic -a measure of between and within-case variability, ranging from 0 to 1 with increasing reliability. The scores were 0.9476 and 0.9164 for Ki-67 and whole mount mitotic counts, respectively, indicating that robust measurements can be obtained without exhaustive sampling of crypts. Moreover, scores from both methods correlated well (Pearson correlation coefficient 0.655, $\mathrm{p}=0.008$ ). There is also concern that individual antibody targets may not display expression that is truly restricted to proliferating cells, and therefore give potentially spurious results. It is reassuring that the immunohistochemical markers used in this study both showed similar results.

Two immunohistochemical markers of cell proliferation, Ki-67 and pHH3, were measured. Colonocyte Ki-67 LI decreased after preparation with Klean-Prep, when compared with unprepared bowel from the same patients (Figure 1A). Despite the consistent change, significance was not achieved ( $\mathrm{p}=0.117$, paired t-test). A decrease was also seen in the number of whole-crypt mitoses with KleanPrep (Figure 1B). Again, the trend was consistent but significance was not achieved $(p=0.118)$. Klean-Prep had no apparent effect on pHH3 diffuse or granular staining, when compared to that seen in the same patients without preparation $(\mathrm{p}=0.519$ and $\mathrm{p}=0.562$, respectively, data not shown).

Picolax had an opposing effect on colonocyte proliferation. Ki-67 LI increased after preparation with Picolax, although this effect was not significant $(\mathrm{p}=0.2778$, Figure 2). Similarly, increased diffuse and granular pHH3 staining was observed with Picolax and reached significance in the granular fraction $(p=0.049, p=0.269$ for positive staining, data not shown). Paired samples from only one patient were available for whole crypt staining; the mean number of mitoses per crypt for this patient were 11.4 and 5.0 for Picolax and unprepared biopsies, respectively.

While obvious and consistent trends are seen in the above analysis, treating the samples as paired for statistical purposes is undermined by the small numbers $(n=4$ for Klean-Prep, $\mathrm{n}=3$ for Picolax) and the unavailability of paired biopsies for all markers tested. All data, paired or otherwise, was therefore redistributed into treatment groups and subjected to analysis of variance (ANOVA) testing. One-way ANOVA revealed significant differences between groups (Ki-67: $\mathrm{p}=0.035$, mitoses: $\mathrm{p}=0.045)$, demonstrating, as for the paired analysis, a decrease in proliferation with Klean-Prep, and an increase with Picolax, when compared to unprepared bowel (Figure 3). Scoring differentially for granular and positive $\mathrm{pHH} 3$ staining showed a similar pattern of response to bowel preparation (data not shown), but statistical significance was reached when the data sets were combined to give a total LI ( $\mathrm{p}=0.038$, Figure 4). Post-hoc analysis revealed a significant difference between Klean-Prep and Picolax on Ki-67 and total pHH3 LI ( $\mathrm{p}=0.019$ and 0.017 , respectively). 

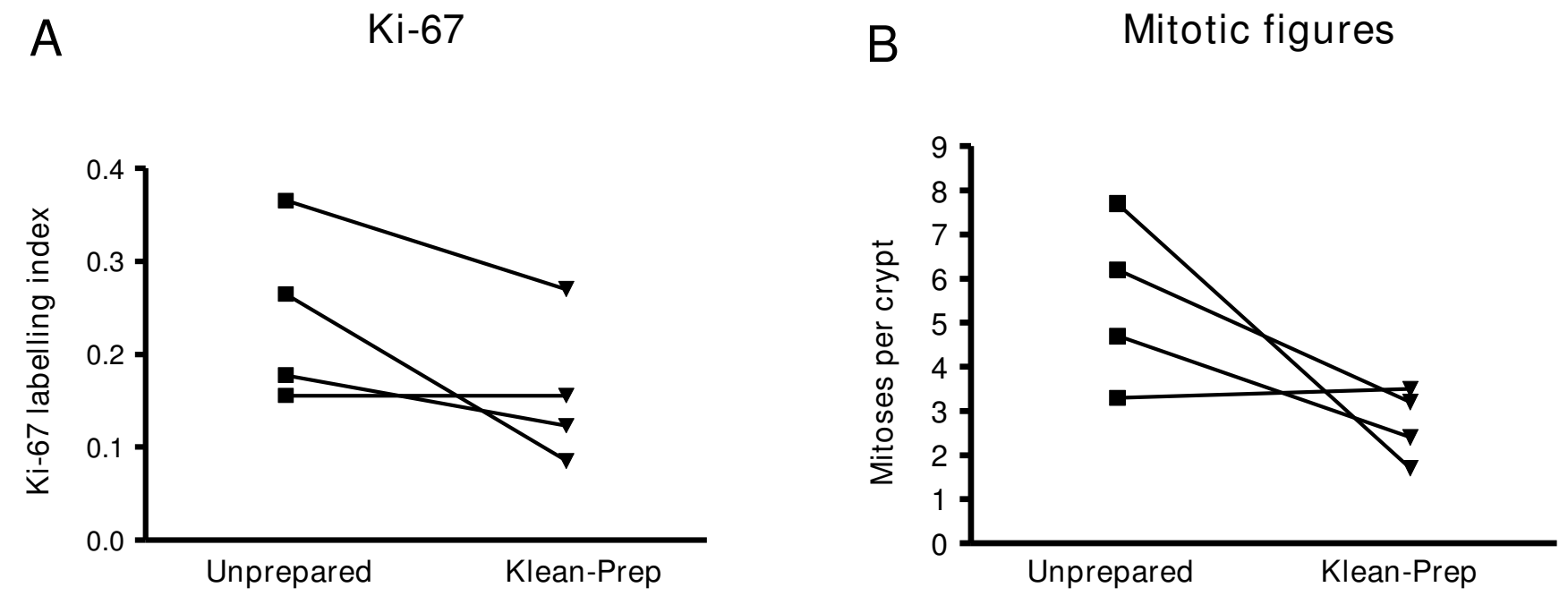

Figure I

Paired t-test analyses of proliferative indices in patients prepared with Klean-Prep. A consistent trend towards suppression of both $\mathrm{Ki}-67(\mathrm{~A})$ and mitoses $(B)$ is seen with Klean-Prep compared to unprepared bowel from the same patients.

This observation may be relevant to the interpretation of proliferation data in several recently published studies. In one study demonstrating no significant difference in Ki67 or whole crypt mitoses between patients with Hereditary non-polyposis colorectal cancer and normal subjects, the authors concluded that crypt cell proliferation is not a suitable discriminative marker for this disease[15]. All patients had been administered Picolax and, as in our study, had Ki-67 LIs in the range 0.3 to 0.4 [16]. A study on the effect of pre-biotic carbohydrates similarly showed no

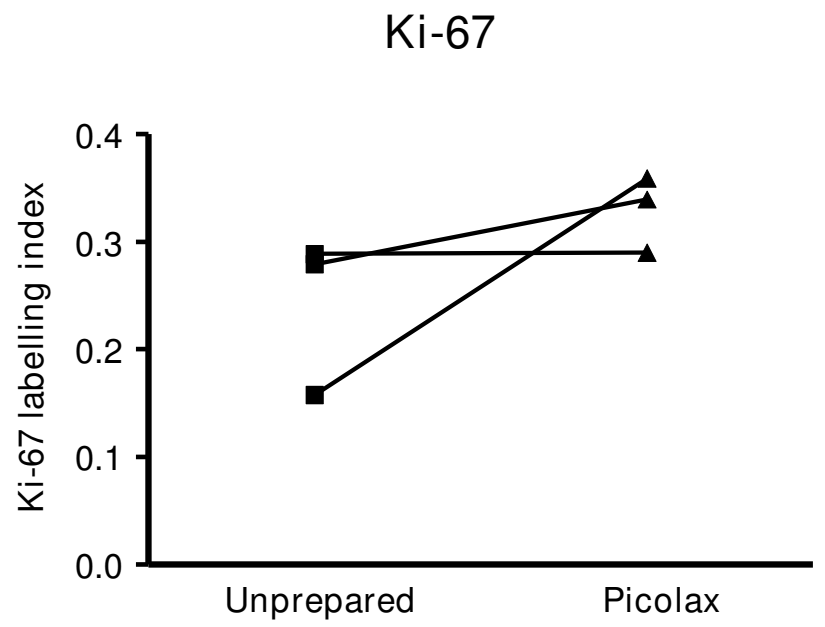

Figure 2

Paired t-test analysis of Ki-67 LI in patients prepared with Picolax. A trend towards elevation of $\mathrm{Ki}-67 \mathrm{LI}$ is seen with Picolax compared to unprepared bowel from the same patients. difference in Ki-67 LI between subjects and controls, reporting indices between 0.3 and 0.4 . Again, all subjects received Picolax. Our evidence suggests that any marginal change in LI in response to disease or intervention in these studies may have been masked by the proliferative effects of Picolax. Equally, data from studies based on subjects prepared with PEG should be interpreted cautiously. No association was found between the PCNA proliferative index (PI) and the likelihood of developing adenoma in one prospective study; the majority of these patients were prepared with PEG[17]. It is possible that PEG suppressed colonocyte proliferation in a group of patients that might be expected to have a higher than normal PI.

\section{Crypt length and cellularity}

No significant differences were seen by t-test analysis between pairs for either Klean-Prep or Picolax $(p=0.766$ and 0.183 , respectively), or between groups by one-way ANOVA $(p=0.437)$. Similarly, there was no significant difference in crypt length between pairs $(p=0.209$ for Klean-prep, $\mathrm{p}=0.827$ for Picolax), or between groups by one-way ANOVA $(\mathrm{p}=0.0786)$ (data not shown). The cellular homeostasis observed despite increased proliferation could be attributed to two factors. Firstly, the interval between bowel preparation and biopsy (typically 12 hours) may have been insufficient for any cells entering cell division to complete the cycle. Secondly, the observed homeostasis may be attributable to increased apoptosis. Our assessment of apoptotic indices recorded very low rates of background apoptosis in all samples, and showed no alteration in response to bowel preparation (data not shown). 
A

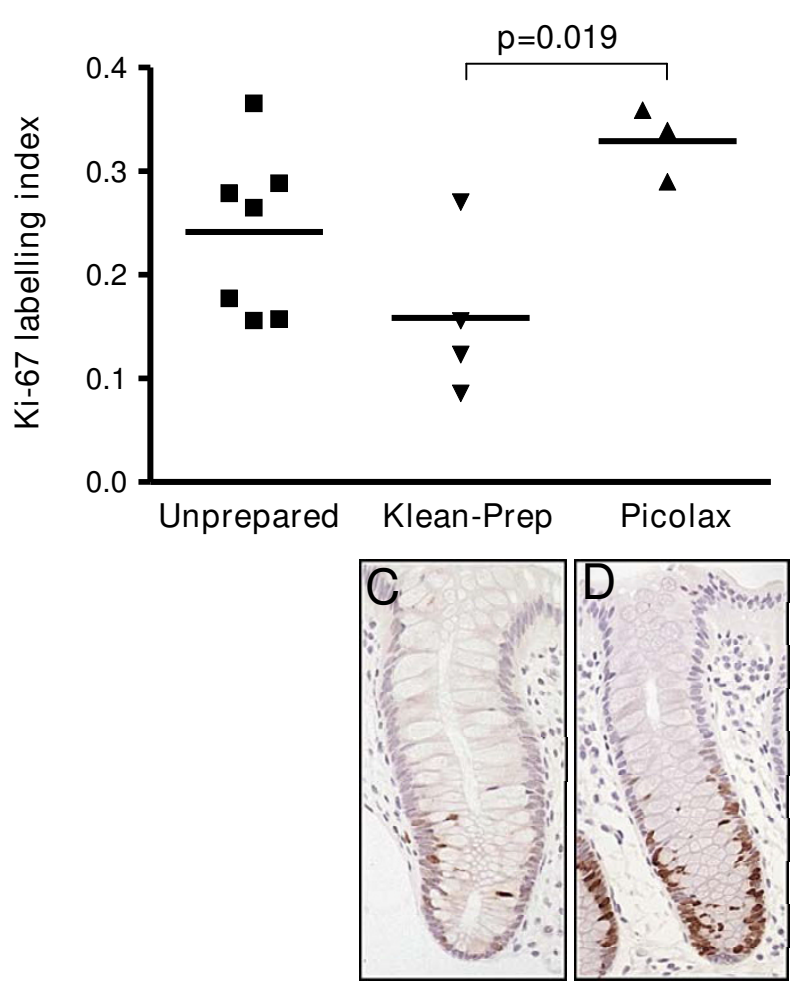

B

Mitotic figures

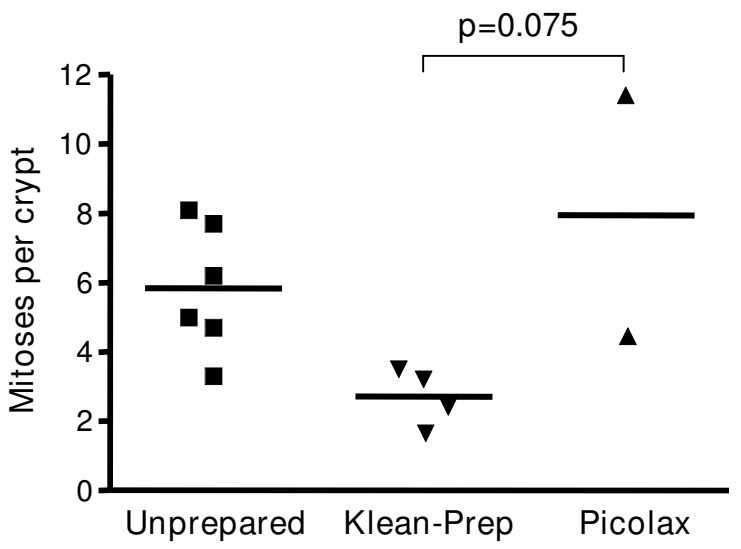

\section{Figure 3}

ANOVA of Ki-67 and mitotic figures in unprepared and prepared bowel. Significant differences were seen between all groups $(p=0.035$ and $p=0.045$ for $\mathrm{Ki}-67(A)$ and mitotic figures $(B)$, respectively), with a marked difference in Ki-67 between Klean-Prep and Picolax $(p=0.019)$. Crypts with a low Ki-67 Ll, from a bowel prepared with Klean-Prep, and with a high LI, from a Picolax prepared bowel, are shown in B and C, respectively.

\section{Limitations of study}

A randomisation bias on clinical grounds cannot be ruled out in the allocation of patients to Klean-Prep or Picolax preparation; this, along with the small sample size, may in part account for the lack of statistical significance seen between prepared patients and unprepared controls. Similarly, whilst patients prepared with Klean-Prep or Picolax fasted prior to colonoscopy, control patients did not, and potential confounding influences of luminal nutrition and luminal workload on proliferation indices should be noted. However, our analyses, and the central finding of this study, show an opposing direction of change between Klean-Prep and Picolax that is consistent for all proliferation measures, and cannot be a feature of starvation. Although the small number of cases and the single sampling site inevitably means that the results should be interpreted with caution, this pilot study has informed our choice of procedural homogeneity for a separate, larger study requiring repeat endoscopies.

\section{Conclusion}

This study demonstrates that Picolax and Klean-Prep have significantly different effects on crypt cell proliferation. Suppression or elevation of proliferation parameters by bowel preparation may mask any effect due to an intervention or disease.

These data underscore the importance of ensuring that all patients participating in studies in which crypt proliferation is an outcome measure are treated with identical bowel preparation. Bowel preparations used in such studies should be reported, and the data interpreted with caution.

\section{Competing interests}

The authors declare that they have no competing interests. 

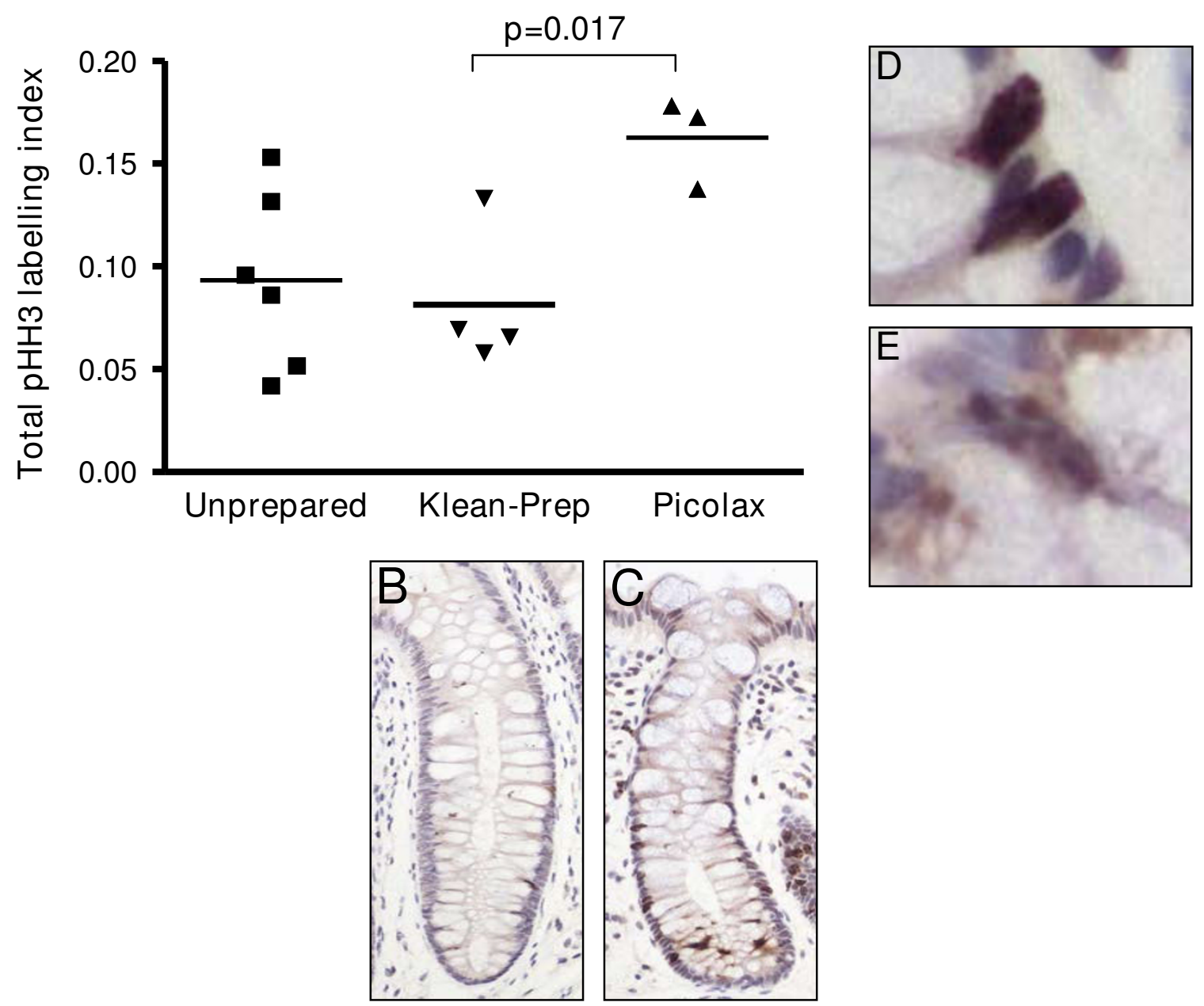

\section{Figure 4}

ANOVA of total (positive and granular) pHH3 in unprepared and prepared bowel. Significant differences were seen between all groups $(p=0.038)$, with a marked difference between Klean-Prep and Picolax $(p=0.017)$. Crypts with a low total $\mathrm{pHH} 3 \mathrm{LI}$, from a bowel prepared with Klean-Prep, and with a high LI, from a Picolax prepared bowel, are shown in B and C, respectively, as well as examples of positive (D) and granular $(E)$ staining.

\section{Authors' contributions}

LJC carried out the analyses and was responsible for drafting the manuscript. JPB contributed to the analyses, performed the statistical analysis and helped to draft the manuscript. EAW participated in the design of the study and assisted with patient recruitment. SR was responsible for patient recruitment and collected biopsy material for the study. BMC conceived the study, participated in its design and helped to draft the manuscript. All authors read and approved the final manuscript.

\section{Acknowledgements}

This study was supported by a Food Standards Agency award (NI20I7).

We acknowledge the assistance of $\mathrm{H}$. Norwood with recruiting.

\section{References}

I. Nelson DB, Barkun AN, Block KP, Burdick JS, Ginsberg GG, Greenwald DA, Kelsey PB, Nakao NL, Slivka A, Smith P, et al.: Technology Status Evaluation report. Colonoscopy preparations. May 200 I. Gastrointestinal endoscopy 200I, 54(6):829-832.

2. Lieberman DA, Ghormley J, Flora K: Effect of oral sodium phosphate colon preparation on serum electrolytes in patients with normal serum creatinine. Gastrointestinal endoscopy 1996, 43(5):467-469.

3. Fordtran JS, Santa Ana CA, Cleveland MvB: A low-sodium solution for gastrointestinal lavage. Gastroenterology 1990, 98(I): I I-I6. 
4. Kastenberg D, Chasen R, Choudhary C, Riff D, Steinberg S, Weiss E, Wruble L: Efficacy and safety of sodium phosphate tablets compared with PEG solution in colon cleansing: two identically designed, randomized, controlled, parallel group, multicenter phase III trials. Gastrointestinal endoscopy 200I, 54(6):705-7|3.

5. Bucher P, Gervaz P, Egger JF, Soravia C, Morel P: Morphologic alterations associated with mechanical bowel preparation before elective colorectal surgery: a randomized trial. Diseases of the colon and rectum 2006, 49(I): 109-II2.

6. Erdogan B, Isiksoy S, Dundar E, Pasaoglu O, Bal C: The effects of sodium phosphate and polyethylene glycol-electrolyte bowel preparation solutions on 2,4,6-trinitrobenzenesulfonic acidinduced colitis in the rat. Exp Toxicol Pathol 2003, 55(2-3):2। 3-220.

7. Fa-Si-Oen PR, Penninckx F: The effect of mechanical bowel preparation on human colonic tissue in elective open colon surgery. Diseases of the colon and rectum 2004, 47(6):948-949.

8. Zwas FR, Cirillo NW, el-Serag HB, Eisen RN: Colonic mucosal abnormalities associated with oral sodium phosphate solution. Gastrointestinal endoscopy 1996, 43(5):463-466.

9. Driman DK, Preiksaitis HG: Colorectal inflammation and increased cell proliferation associated with oral sodium phosphate bowel preparation solution. Human pathology 1998, 29(9):972-978.

10. Yang K, Fan K, Mengs U, Lipkin M: Effects of sennosides and nonanthranoid laxatives on cytochemistry of epithelial cells in rat colon. Pharmacology 1993, 47(Suppl I):196-204.

II. Geboes K, Nijs G, Mengs U, Geboes KP, Van Damme A, de Witte P: Effects of 'contact laxatives' on intestinal and colonic epithelial cell proliferation. Pharmacology 1993, 47(Suppl I): I87-195.

12. Toyoda K, Nishikawa A, Furukawa F, Kawanishi T, Hayashi Y, Takahashi M: Cell proliferation induced by laxatives and related compounds in the rat intestine. Cancer Lett 1994, 83(I-2):43-49.

13. Hendzel MJ, Wei Y, Mancini MA, Van Hooser A, Ranalli T, Brinkley BR, Bazett-Jones DP, Allis CD: Mitosis-specific phosphorylation of histone $\mathrm{H} 3$ initiates primarily within pericentromeric heterochromatin during $\mathbf{G 2}$ and spreads in an ordered fashion coincident with mitotic chromosome condensation. Chromosoma 1997, 106(6):348-360.

14. Goodlad RA, Levi S, Lee CY, Mandir N, Hodgson H, Wright NA: Morphometry and cell proliferation in endoscopic biopsies: evaluation of a technique. Gastroenterology 1991, I0I(5): |235-|24|.

15. Green SE, Chapman P, Burn J, Burt AD, Bennett M, Appleton DR, Varma JS, Mathers JC: Colonic epithelial cell proliferation in hereditary non-polyposis colorectal cancer. Gut 1998 43(I):85-92.

16. Langlands SJ, Hopkins MJ, Coleman N, Cummings JH: Prebiotic carbohydrates modify the mucosa associated microflora of the human large bowel. Gut 2004, 53(II):1610-1616.

17. Sandler RS, Baron JA, Tosteson TD, Mandel JS, Haile RW: Rectal mucosal proliferation and risk of colorectal adenomas: results from a randomized controlled trial. Cancer Epidemiol Biomarkers Prev 2000, 9(7):653-656.

\section{Pre-publication history}

The pre-publication history for this paper can be accessed here:

http://www.biomedcentral.com/1471-230X/8/54/pre pub

\section{Publish with Biomed Central and every scientist can read your work free of charge}

"BioMed Central will be the most significant development for disseminating the results of biomedical research in our lifetime. "

Sir Paul Nurse, Cancer Research UK

Your research papers will be:

- available free of charge to the entire biomedical community

- peer reviewed and published immediately upon acceptance

- cited in PubMed and archived on PubMed Central

- yours - you keep the copyright

Submit your manuscript here:

http://www.biomedcentral.com/info/publishing_adv.asp
BioMedcentral 